\title{
CLINICAL AND RADIOGRAPHIC EVALUATION OF BONE AUGMENTATION AROUND DENTAL IMPLANT USING DIFFERENT BONE GRAFT MATERIALS
}

\author{
Mohammad S Tawakol*, Mohammed M Khedr ${ }^{* *}$, Abdelfattah M Amer ${ }^{* * *}$
}

\begin{abstract}
Background: The present study evaluated the augmented bone around a dental implant utilizing synthetic bone (Sinoss Graft) or xenograft bone (Tutobone Graft) materials. Methods: A group of patients were divided into two groups, based on the graft material used. Group I Patients were treated by placing two dental implants, with guided bone regeneration using synthetic bone material. (Sinoss Graft) and Group II Patients were treated by placing two dental implants, with guided bone regeneration using xenograft bone material (Tutobone Graft). Results: GTR + Xenograft group recorded a higher Osstell measurement than GTR + synthetic bone group. In GTR+ synthetic bone or GTR + xenograft groups; there was a statistically significant different. GTR + xenograft showed a higher bone density than GTR+ synthetic bone group. Conclusion: Treatment of osseous defects using Xenografts in combination with GTR, generally, has significant better clinical outcome.
\end{abstract}

\section{INTRODUCTION}

It is well documented that, tooth extraction has been followed by a reduction of buccolingual as well as apicocoronal dimension of the alveolar ridge at the edentulous area ${ }^{(1,2)}$. The use of guided bone regeneration $(\mathrm{GBR})$ in treatment of ridge deficiencies was considered a safe and predictable treatment modality ${ }^{(3,4)}$. It should be emphasized that, augmentation of the resorbed alveolar crest can be achieved, for example, with onlay bone grafts, membrane techniques, bone distraction and bone splitting. Bone grafting and guided bone regeneration can increase the width and, to some extent, the height of the alveolar bone. Lateral widening, but not vertical augmentation, is possible with a crestal split technique. A feature common to all these treatments is that they are technique sensitive. This is why the success rates differ in various follow-up studies and no prospective study on randomized material exists to estimate the benefits of various bone augmentation procedures ${ }^{(5,6)}$.

The ridge width affects many of the final restoration's parameters, which can easily be overlooked without careful preoperative planning. Edentulous alveolar ridges less than $5 \mathrm{~mm}$ in width require horizontal augmentation or horizontal expansion of the alveolar bone for the placement of the endosseous implants in order to produce the necessary quantity of bone at least $2 \mathrm{~mm}$ around the implants and to guarantee long-term osseointegration $^{(7)}$.

In case of in-adequate ridge width, several problems may occur as leaving a thin labial bone plate at the implant placement time that may cause implant exposure after ridge resorption, a labial dehiscence of bone may contribute to future

\footnotetext{
* Dentist: Ministry of Health

** Professor and Head of Oral Medicine, Periodontology, Oral Diagnosis and Radiology Department, Faculty of Dental Medicine (Boys, Cairo), Al-Azhar University

*** Professor of Oral Medicine, Periodontology, Oral Diagnosis and Radiology Department, Faculty of Dental Medicine (Boys, Cairo), Al-Azhar University
} 
impactites or an unaesthetic metal showing through gingival, or undercuts found on labial alveolar bone give rise to off-axis loads and less perfect emergence profile. In an attempt to overcome the above-mentioned problems, the deficient sites are either grafted or enhanced by different means ${ }^{(7)}$. In these situations, it was felt that performing a study on bone augmentation around dental implant utilizing different bone graft materials may extend further the possibility of inserting dental implants into narrow ridges, through bone augmentation by grafting materials. Additionally, it will be of interest to examine the efficacy of this procedure clinically and radiographically.

\section{MATERIALS AND METHODS}

A group of patients were selected from Outpatient Clinic, Department of Oral Medicine, Periodontology, Oral Diagnosis and Oral Radiology, Faculty of Dental Medicine, (Boys, Cairo),
Al-Azhar University. Patients were divided into two groups, based on the graft material used. Group I Patients were treated by placing two dental implants, with guided bone regeneration using synthetic bone material. (Sinoss Graft) and Group II Patients were treated by placing two dental implants, with guided bone regeneration using xenograft bone material (Tutobone Graft).

Surgery was performed under local anesthesia and strict aseptic conditions. Flap was reflected, Sequential drilling with copious irrigation was carried out till the desired dimensions were achieved depending on the selected implant. Space between the implant and walls of the prepared socket were measured and bone graft was placed. Collagen membrane was placed and suturing of the flap was done. Patients were evaluated clinically at 3,6, 9 and 12 months postoperatively and radiographs were recorded at baseline (BL) and 12 months postoperatively.

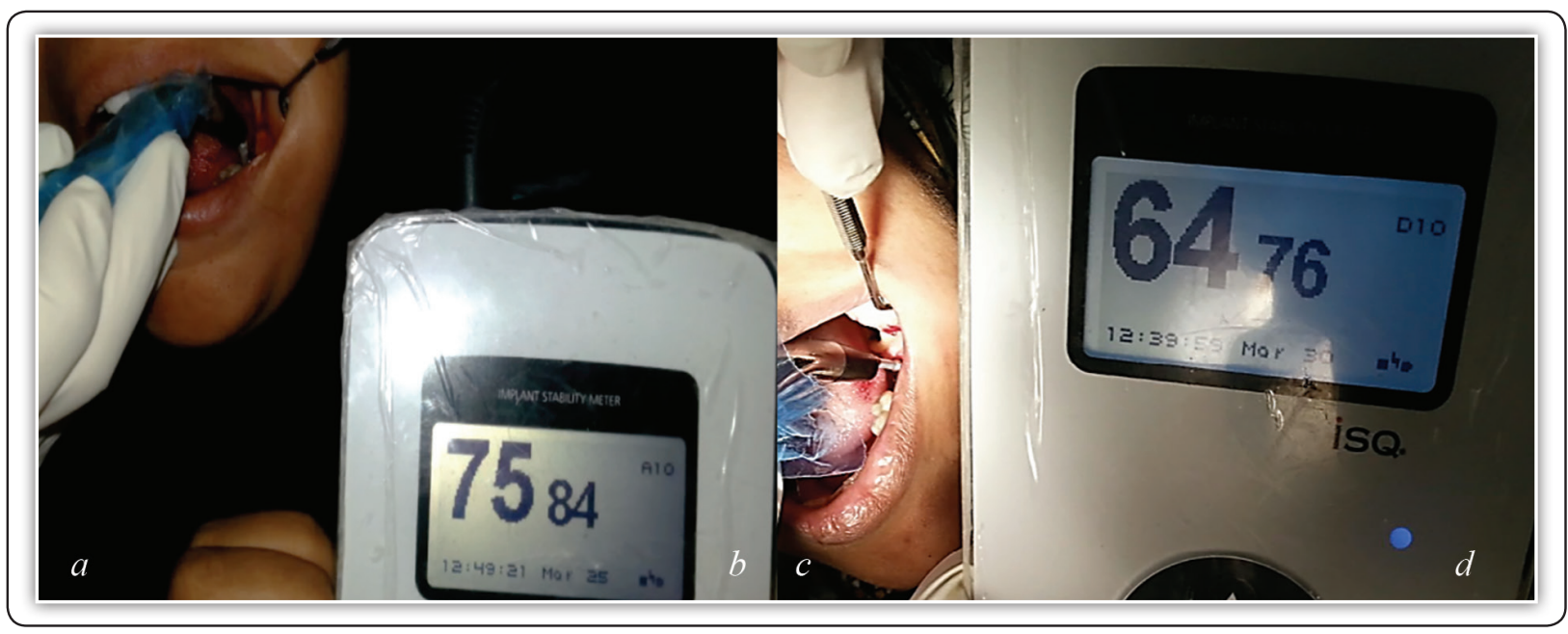

FIG (1) a. Osstell measurement after 3 months of implant placement with guided bone regeneration using synthetic bone material, b. Osstell measurement after 12 months of implant placement with guided bone regeneration using synthetic bone material, c. Osstell measurement after 3 months of implant placement with guided bone regeneration using xenograft bone material, d. Osstell measurement after 12 months of implant placement with guided bone regeneration using xenograft bone material. 


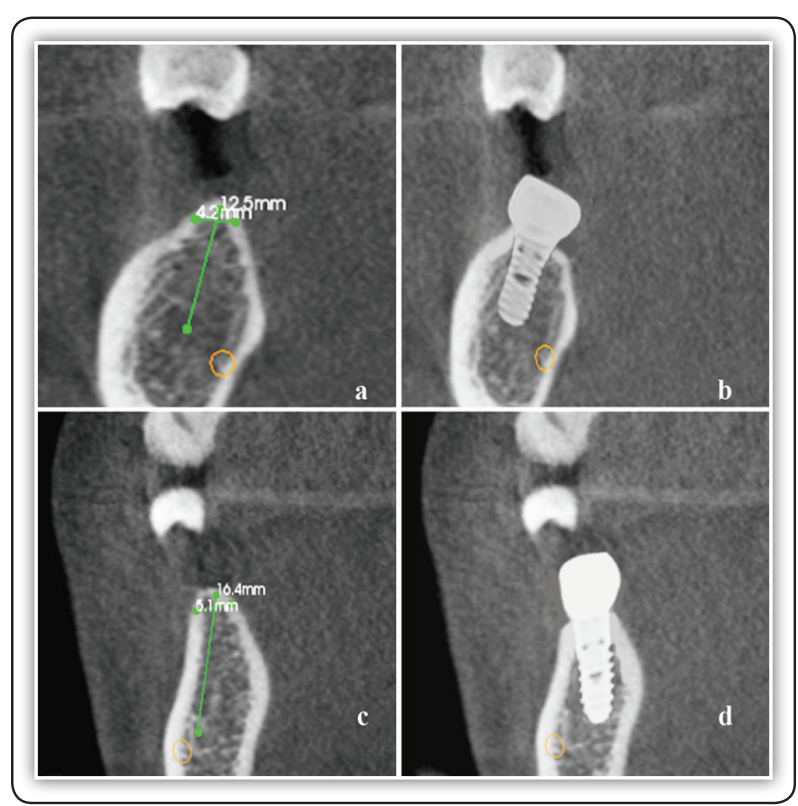

\section{RESULTS}

The Comparison between the two groups regarding to Osstell measurements and bone density at different follow up periods:

At 3 months; there was non-statistically significant change in mean Osstell. At 6, 9, 12
FIG (2) a. Sectional tracing for implant simulation replacing lower left first premolar, showing buccal bone defect, b. Radiograph showing dental implant replacing lower left first premolar after 12 months of placement and increased in thickness of buccal cortex, c. Sectional tracing and implant simulation replacing lower left first molar, showing buccal bone defect, d. Radiograph showing dental implant replacing lower left first molar after 12 months of placement and increased in thickness of buccal cortex.

TABLE (1): Comparison between the two groups regarding Osstell measurements and bone density

\begin{tabular}{|c|c|c|c|c|c|c|}
\hline \multirow{2}{*}{} & \multicolumn{2}{|c|}{ GTR + synthetic bone } & \multicolumn{2}{c|}{ GTR + Xenograft } & \multirow{2}{*}{$\mathrm{t}$} & \multirow{2}{*}{ p } \\
\cline { 2 - 6 } & Mean & $\mathbf{\pm S D}$ & Mean & $\mathbf{\pm S D}$ & \\
\hline \multicolumn{7}{|c|}{ Osstell } \\
\hline 3 months & 68.33 & 1.97 & 69.83 & 0.41 & 1.830 & 0.122 \\
\hline 6 months & 68.50 & 1.05 & 76.0 & 0.0 & $17.516^{*}$ & $<0.001^{*}$ \\
\hline 9 months & 74.50 & 1.76 & 82.67 & 2.80 & $6.041^{*}$ & $<0.001^{*}$ \\
\hline 12 months & 71.83 & 8.01 & 87.17 & 5.53 & $3.859^{*}$ & $0.003^{*}$ \\
\hline \multicolumn{7}{|c|}{ Bone density } \\
\hline Baseline & 246.67 & 35.34 & 262.50 & 20.71 & 2.143 & 0.058 \\
\hline 12 months & 721.67 & 9.05 & 765.83 & 38.39 & 2.122 & 0.060 \\
\hline
\end{tabular}

$t, p: t$ and $p$ values for Student t-test for comparing between the two groups 


\section{DISCUSSION}

The use of a dental implant has become a common treatment and an important part of the modern dental practice. In this respect, an implantation into fresh extraction sockets has been recommended as a means to minimize bone loss as well as to shorten the prosthetic treatment time. ${ }^{(6)}$ However, the residual bone defects, between the implant neck and the residual bone walls, may cause cell migration from the connective and epithelial tissue into the defect area, possibly preventing the osseointegration ${ }^{(8)}$, which may jeopardize the success of immediate implant procedures. It has been postulated that when the size of horizontal gap surrounding immediately placed implants exceeds the threshold of 1 to $2 \mathrm{~mm}$, bone graft procedures might be recommended to reduce peri-implant bone resorption and improve the esthetic outcome of the soft tissue. ${ }^{(9)}$

Autogenous bone grafts are considered the gold standard mean of grafting, because of their osteogenic, osteoconductive, and Osteoinductive properties ${ }^{(10)}$. However, searching for a bone graft substitute should be continued to overcome some of disadvantages associated with the use of autogenous bone grafts as morbidity of donor sites, longer surgical time, and higher costs. Xenogeneic bone grafts consist of de-protenized cancellous skeletal bone tissue that is harvested from one species and transferred to the recipient site of another species. One of the graft materials in this respect is the inorganic bovine bone (IBB), which is a xenograft bone with same chemical and physical properties of human bone. IBB may facilitate bone repair as histological finding, showed newly formed bone in direct contact with IBB particles. ${ }^{(11)}$ The inorganic component of the xenograft not only serves as natural structural matrix for new bone formation, but also results in an excellent source of calcium, which is essential for bone formation. Furthermore, xenograft is used in combination with growth factors and or allografts to simulate the autogenous bone ${ }^{(12)}$.
The present study evaluated augmented bone around a dental implant utilizing synthetic bone (Sinoss Graft) or xenograft bone (Tutobone Graft) materials to fill the horizontal gap surrounding the implants. There is no control group in which bone graft procedure would not be carried out was not included in this study. Osseointegration and peri-implant tissue modeling following implant placement in fresh extraction sockets was evaluated, and it was found that in the absence of bone graft, the dimensions of both the buccal and the lingual bone walls around the implant were reduced; even the osseointegration could be in part lost following bone modeling ${ }^{(13)}$. In the present study, at $6,9,12$ months, the difference was statistically significant. GTR + Xenograft group recorded a higher stability (Osstell) measurement than GTR + synthetic bone group. The xenografts filled the horizontal gap between the bony walls and the implants. The stability of an implant is secondary and tertiary. Secondary stability is a function of repair process beginning after implant placement. It is biologically determined and associated mainly with cancellous bone and cancellous bone events. The tertiary stability is the maintenance of osseointegration ${ }^{(14)}$. Various tests and methods for implant stability assessment are applied. Osstell is a non-invasive diagnosis technique that uses a piezoelectric transducer, which uses magnetic pulses within a specific frequency meant to make the implant vibrate. Implant resistance to vibration is measured by the device and transformed into an ISQ value (implant stability quotient - within a 0-100 scale, 100 being maximum implant stability). The influence of grafting procedure on primary stability of immediately placed implants, nevertheless, has not been given enough attention.

It has been proven that alveolar bone remodeling appeared progressively active following tooth extraction; the horizontal resorption of the buccal alveolar dimension amounted to about $56 \%$ at 4 months after tooth loss ${ }^{(15)}$. Xenografts play an important part in alveolar bone preservation and can maintain the dimensions of the extraction socket, 
as well as encourage osteoconduction and space maintenance. ${ }^{(16)}$

Studies ${ }^{(17,18)}$ reported that after the third post implantation month, the measured Osstell was considerably higher both vs primary stability and one-month stability values. This is associated to alveolar bone remodeling events. The results of present study lend support to these results. The difference was still present after 6 months and was also statistically significant in the present study.

In GTR + synthetic bone or GTR + xenograft groups; there was a statistically significant change in mean Bone density through all periods. GTR + xenograft showed a higher bone density than GTR +synthetic bone group. The efficacy of a xenograft as an alveolar bone graft material may be the result of a combination of factors: its osteoconductive capacity, the increase of mineral content in the grafted area necessary for bone formation and its density in order to provide stability to the graft and to persist for many months. Xenografts were bone minerals derived from animals where the organic components were eliminated in order to reduce the recipient immune reactions ${ }^{(19)}$.

Alloplastic synthetic biomaterials were developed to overcome the disadvantages of autografts and Xenografts and are fabricated in various forms with varying physicochemical properties and can be both degradable and non-degradable. Allopath are usually osteoconductive without any Osteoinductive or osteogenic potential on their own and have been used extensively for periodontal regeneration. ${ }^{(20)}$

Evaluating bone density has been one of the most important parameters to quantify bone quality, as it is thought to be a major determinant of primary stability. In other words, primary implant stability is dependent on not only the thickness of the bone into which the implant is placed, but also on bone density. the level of bone density at the implant site could be of utmost importance as it is related with failure rates and primary stability. ${ }^{(21)}$

\section{CONCLUSION}

Within the limitation of the present study, the following conclusion can be drawn,

1. Xenografts can be considered regenerative and biocompatible grafting material; obtained results extended further this fact.

2. Treatment of osseous defects using Xenografts in combination with GTR, generally, has significant better clinical outcome.

3. Dental implant, with guided bone regeneration using xenograft bone showed high implant stability over that obtained with using synthetic bone.

\section{REFERENCES}

1. Schropp L, Wenzel A, Kostopoulos L. Bone healing and soft tissue contour changes following single-tooth extraction: a clinical and radiographic 12-month prospective study. Int J Perio Restor. Dent 2003; 23:313-23

2. Abrams H, Kopczyk R, Kaplan A. Incidence of anterior ridge deformities in partially edentulous patients. J Prosthet Dent 1987; 57:191-4.

3. Dahlin C, Simion M, Hatano N. Long-term follow-up on soft and hard tissue levels following guided bone regeneration treatment in combination with a xenogeneic filling material: a 5-year prospective clinical study. Clin Implant Dent Relat Res 2010; 12:263-70.

4. Benić G, Jung R, Siegenthaler D. Clinical and radiographic comparison of implants in regenerated or nativebone: 5-year results. Clin Oral Implants Res 2009; 20:507-13.

5. Ady P, Marius S. Modern Methods in Augmentation Surgery; Int Mag of Oral Implantology. 2004; 2:12-4.

6. Ady P. Long term success with sinus elevation criteria and parameters; Int Mag of Oral Implantology. 2004; 3:2-5.

7. Schipani A, Bruschini G, Celestina G: The edentulous ridge $\mathrm{Vol}$ expansion technique; A five year study; Int $\mathrm{J}$ Perio Restor. Dent.1994;14: 451-9.

8. Landsberg C. Socket seal surgery combined with immediate implant placement: a novel approach for single tooth replacement. Int J Perio Restor. Dent. 1997; 17:141-9.

9. Harel N, Moses O, Palti A, Ormianer Z. Long-term results of implants immediately placed into extraction sockets grafted with $\beta$-tricalcium phosphate: a retrospective study. J Oral Maxillofac Surg. 2013; 71:e63-8 
10. Rajan G, Fornaro J, Trentz O, Zellweger R. Cancellous allograft versus autologous bone grafting for repair of comminuted distal radius fractures: A prospective, randomized trial. J Trauma. 2006; 60:1322-9.

11. Dias R, Sehn F, Santos T, Silva E, Chaushu G. Corticocancellous fresh-frozen allograft bone blocks for augmenting atrophied posterior mandibles in humans. Clin Oral Implants Res. 2014; 5: 188-210.

12. Richardson C, Mellonig J, Brunsvold M, McDonnell H, Cochran D. Clinical evaluation of Bio-Oss: A bovine-derived xenograft for the treatment of periodontal osseous defects in humans. J Clin Perio 1999; 26 :421-8.

13. Araújo M, Wennström J, Lindhe J. Modeling of the buccal and lingual bone walls of fresh extraction sites following implant installation. Clin Oral Implants Res. 2006; 17:606-14.

14. Atsumi M, Park S, Wang H. Methods used to assess implant stability: current status. Int J of Oral and Maxillofac Implant. 2007; 22:743-54.

15. Botticelli D, Berglundh T, Lindhe J. Hard-tissue alterations following immediate implant placement in extraction sites. J Clin Perio .2004; 31:820-8
16. Barone A, Aldini N, Fini M, Giardino R, Calvo J, Covani $U$. Xenograft versus extraction alone for ridge preservation after tooth removal: a clinical and histomorphometric study. J Perio. 2008; 79:1370-7.

17. Akca, K., Chang T, Tekdemir L, Fanuscu M. Biomechanical aspects of initial intraosseous stability and implant design: a quantitative micromorphometric analysis Clin Oral Implant Res. 2006; 17: 465-72.

18. Maria F, Sergio A , Patricia M , Jose L. Calvo-Guirado 1 and Piedad N. De Aza. Implant Stability of Biological Hydroxyapatites Used in Dentistry. Materials 2017, 10, 644

19. Figueiredo A. Inflammatory reaction post implantation of bone graft materials. Experimental Pathology and Health Sciences 2012; 1: 15-8.

20. Shetty V, Han T. Alloplastic materials in reconstructive periodontal surgery. Dent Clin N Am. 1991;35:521-30.

21. Iezzi G, Scarano A, Di Stefano D, Arosio P, Doi K, Ricci $\mathrm{L}$, et al. Correlation between the bone density recorded by a computerized implant motor and by a histomorphometric analysis: A preliminary in vitro study on bovine ribs. Clin. Implant. Dent. Relat. Res. 2015; 17: 35-44. 